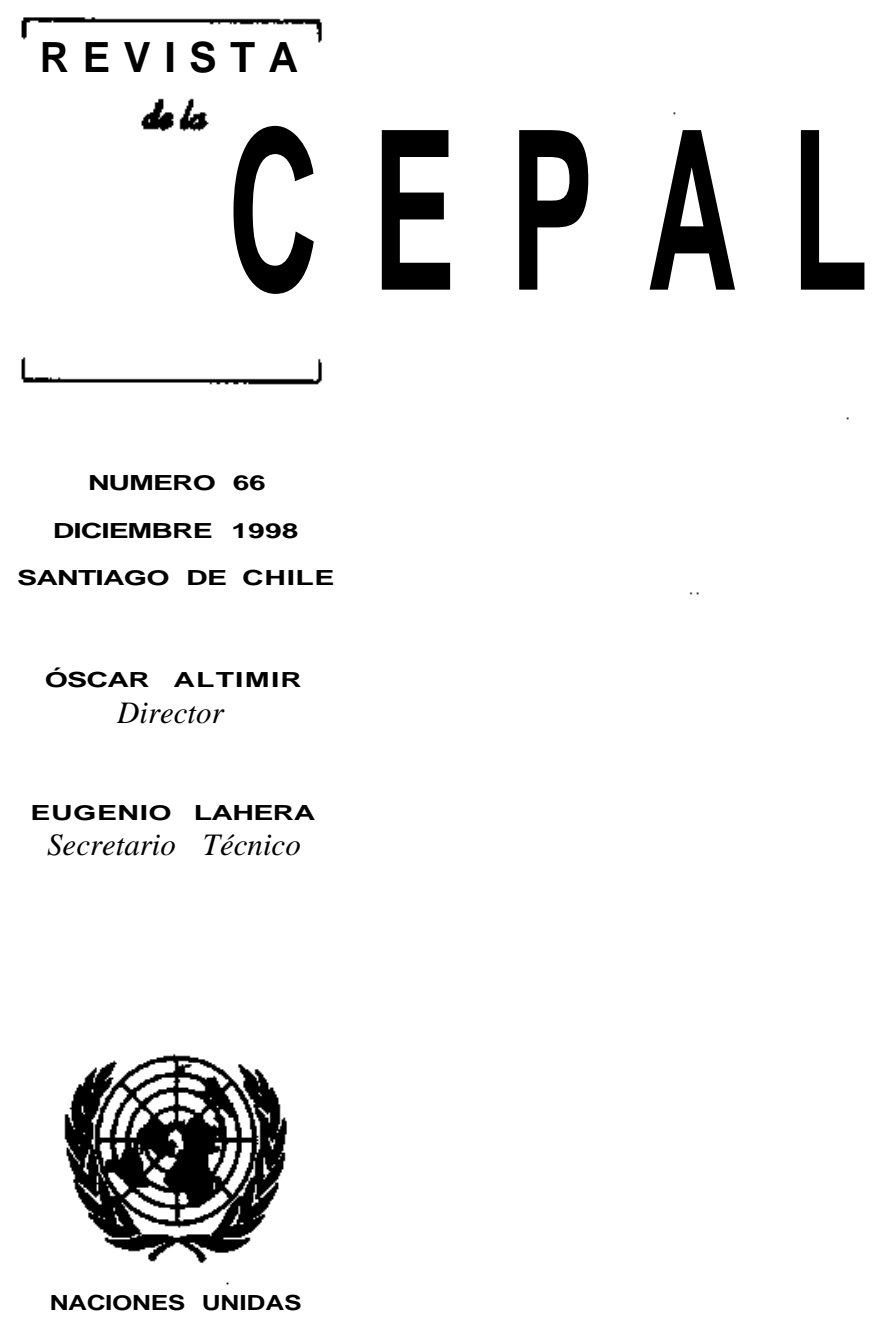


Más allá del Consenso de Washington: una visión desde la CEPAL

José Antonio Ocampo

La economía de Cuba

David Ibarra y Jorge Máttar

La educación en América Latina: la demanda y la distribución importan

Nancy Birdsall, Juan Luis Londoño y Lesley O'Connell

Determinantes de la desigualdad entre los hogares urbanos

Luis Felipe Jiménez L, y Nora Ruedi A.

Los compromisos de gestión en salud de Costa Rica con una perspectiva comparativa

Ana Sojo

Una estrategia de desarrollo a partir de complejos productivos en torno a los recursos naturales

Joseph Ramos

Grandes empresas y grupos industriales latinoamericanos

Celso Garrido y Wilson Peres

Entre el control político y la eficiencia: evolución de los derechos de propiedad agraria en México

Gustavo Gordillo, Alain de Janvry y Elizabeth Sadoulet

Los aranceles y el Plan Real de Brasil

Renato Baumann, Josefina Rivero y Yohana Zavattiero

Publicaciones recientes de la CEPAL 


\section{Los aranceles y el Plan Real de Brasil}

Renato Baumann Josefina Rivero Yohana Zavattiero

Oficina de la CEPAL en Brasilia
En este artículo se analiza la lógica económica de la política arancelaria de Brasil durante los primeros dos años del Plan Real. Con este fin se estudian las modificaciones a las tasas del impuesto de importación para todos los productos comercializados. El proceso de reforma arancelaria de Brasil se inició en 1988, después de treinta años de vigencia de la Ley de Aranceles, y correspondió a una profundización marcada del proceso de apertura comercial con la definición de una trayectoria decreciente para las tasas arancelarias, que se intensificó a partir de 1990. El Plan Real se inició en julio de 1994 y tuvo efectos múltiples sobre la política de importaciones. El proceso de liberalización comercial alcanzó su punto máximo en el primer trimestre de 1995, reflejado en el nivel medio de las tasas y el grado de dispersión de su estructura. Desde entonces ocurrieron diversos episodios de aumento de tasas, en forma diferenciada por sectores, los que aumentaron el grado de dispersión de la estructura arancelaria. Si se excluyen dos sectores con tratamientos específicos — petróleo y automóviles-, se observa una relación directa entre el valor importado y el nivel del arancel nominal, así como variaciones pronunciadas a lo largo del período. Por otra parte, el hecho de que para algunos sectores la tasa ponderada por el valor de las importaciones fue inferior por meses a la tasa media indica que hay margen para reexaminar los niveles arancelarios en algunos casos. El artículo concluye con algunas reflexiones sobre el desempeño de la política de importaciones. 


\section{I}

\section{Introducción}

La economía brasileña se cita con frecuencia en la literatura sobre política comercial externa. Han sido analizados también en diversos estudios la multiplicidad de instrumentos adoptados como barreras a las importaciones que compiten con la producción interna (sobre todo en los decenios de 1960 y 1980), los mecanismos empleados en la práctica para sortear esas barreras aplicando regímenes especiales de importación, los diversos incentivos para las exportaciones, el proceso de definición de los gravámenes a la importación y la preocupación por neutralizar los eventuales sesgos de la política comercial.

No hace mucho, el país experimentó un proceso de reforma de la estructura arancelaria que se inició en 1988, luego de 30 años de vigencia de la llamada Ley Arancelaria, que sufrió algunas modificaciones en 1989 y muchas más en 1990.

La adopción del Plan Real —en julio de 1994tuvo para la política de importaciones múltiples efectos. Los efectos-ingreso y precio vinculados con un programa exitoso de estabilización constituyen un estímulo natural para la demanda de productos importados. Además, la preocupación por viabilizar el acceso a productos externos más baratos cumplía la doble función de presionar a los grupos con una posición oligopolista en el mercado nacional e inducir una mayor eficiencia del aparato productivo.

A las preocupaciones por la estabilidad de precios se sumó una cuestión coyuntural dada por la proximi- dad de la fecha prevista para la entrada en vigor de la estructura arancelaria negociada con los demás socios del Mercosur: el Arancel Externo Común estaba programado para entrar en vigencia a partir del $1^{\circ}$ de enero de 1995. Por lo tanto, el segundo semestre de 1994 estuvo marcado por continuas variaciones de las tasas del gravamen sobre las importaciones. ${ }^{1}$

Este tema ya lo trató Kume (1996) con bastante competencia, en tanto que Nonnemberg (1996) analizó otras medidas de política correlacionadas; el primero estima la evolución — desde 1988 - de la tasa de protección efectiva otorgada a los diversos sectores productores, y el segundo destaca la importancia de los movimientos de arbitraje financiero como estímulo para financiar las importaciones. ${ }^{2}$

En el presente artículo procuramos identificar la lógica económica de la política arancelaria a partir del examen de cada una de las diversas modificaciones de las tasas de los gravámenes a la importación para todos los productos comercializados. El período cubierto comprende desde julio de 1994, cuando se inició el Plan Real, hasta fines de septiembre de 1996, último mes con informaciones disponibles al momento de preparar este trabajo. Se consideraron todos los decretos y resoluciones que afectaban las tasas del gravamen, lo que permitió describir la evolución de la política nacional de importaciones en los primeros dos años del Plan Real. El anexo metodológico muestra cómo se procesaron los datos primarios.

\section{II}

\section{Evolución de la estructura arancelaria}

A lo largo de la presente década, la política comercial externa brasileña siguió una trayectoria de reducción de las barreras comerciales, lo que se reflejó en el

D El presente trabajo no podría haberse realizado sin el competente apoyo computacional de Decio Fialho de la CEPAL. Igual importancia revistió la colaboración de André Bauer en el procesamiento de datos. aumento correspondiente del valor de las importaciones (cuadro 1).

\footnotetext{
1 Cabe señalar que las prioridades del Plan Real -adoptado a partir del $1^{\circ}$ de julio de 1994- también tuvieron una gran influencia en la política nacional de aproximación al Arancel Externo Común.

2 Para una descripción relativamente completa del período anterior a la adopción del Plan Real (1958-1993), véase Pinheiro y Almeida (1995).
} 
CUADRO )

Brasil: Gravámenes a la importación y valor importado, 1990-1996

\begin{tabular}{lcc}
\hline Año & $\begin{array}{c}\text { Tasa media simple } \\
\text { (porcentual) }\end{array}$ & $\begin{array}{c}\text { Valor de las importaciones } \\
\text { (en millones de dólares fob) }\end{array}$ \\
\hline 1990 & 32.12 & 20661 \\
1991 & 25.19 & 12042 \\
1992 & 20.78 & 20554 \\
1993 & 16.49 & 22797 \\
1994 & 13.97 & 33106 \\
1995 & 13.07 & 49263 \\
1996 & & 49619 \\
(enero/septiembre) & 13.10 & 49 \\
\hline
\end{tabular}

Fuente; Estimaciones proporcionadas por H. Kume y elaboración propia a partir de datos primarios.

Interesa observar en el cuadro 1 que aunque la tasa del gravamen tiende a bajar sostenidamente (salvo una pequeña inflexión en el último año) —y que a eso corresponde un aumento del valor total de las importaciones - hay una diferencia de ritmo que no debería pasar inadvertida. Entre 1990 y 1993 la tasa media simple se redujo a la mitad, mientras que los aumentos más notorios de las importaciones ocurrieron en el período más reciente. Además, hay una estabilización relativa de la tasa del gravamen a las importaciones desde 1994, pero -como se verá más adelante- en realidad esa estabilidad oculta un conjunto variado de reducciones y alzas simultáneas de tasas para diversos productos.

Para explicar la diferencia del ritmo de variación del impuesto y el mayor valor de las importaciones, hay que considerar otros elementos atinentes, como la política cambiaria, el efecto-ingreso de la demanda de productos importados, la existencia de regímenes especiales de importación e incluso un aspecto que suele despreciarse en buena parte de los análisis: a saber, el proceso mismo de familiarización - de los consumidores - con productos fabricados en el exterior que no eran obtenibles en el mercado nacional durante décadas. Este último es un proceso lento que entraña la creación y consolidación de canales de comercialización interna de los productos importados, y explica, por cierto, al menos en parte, el desfase cronológico de la respuesta de la demanda de esos productos.

\section{Características generales del proceso de aper- tura}

\section{a) Productos afectados y aranceles medios}

$\mathrm{El}$ primer aspecto que debe destacarse al analizar la política de importaciones en los dos primeros años del Plan Real es que las variaciones ${ }^{3}$ de las tasas del gravamen a las importaciones afectaron al $83 \%$ del total de la lista y que $28 \%$ de los productos afectados sufrieron dos o más modificaciones de las tasas en el período comprendido entre julio de 1994 y septiembre de 1996. En otras palabras, se trata indiscutiblemente de un momento de notoria redefinición de las relaciones comerciales con el resto del mundo.

Las cifras del cuadro 2 indican que en ese período la estructura del gravamen a las importaciones estuvo sujeta a una fuerte intervención. Incluso diversos productos específicos (a los diez dígitos de la clasificación) sufrieron varias alteraciones de la tasa nominal. En 148 productos hubo cinco modificaciones o más, lo que —en el intervalo de 27 meses — puede haberse convertido en un elemento perturbador de la formación de expectativas por parte de los agentes económicos.

Surge un cuadro semejante de diferencias de! ritmo de la apertura comercial y el de desempeño importador si se considera el mismo período analizado aquí (julio de 1994 a septiembre de 1996) (cuadro 3 y gráfico 1)

En ese período hubo un momento inicial (en los últimos dos trimestres de 1994) de notoria reducción del gravamen sobre las importaciones que — sumada a otros factores - tuvo como consecuencia un aumento significativo del valor importado. Ya desde comienzos de 1995 se observa una leve alza de la tasa media simple, que a partir del segundo trimestre alcanza un nivel (de alrededor de 13\%) que se ha mantenido desde entonces. El valor de las importaciones trimestra-

CUADRO 2

Brasll: Incidencia de las variaciones del gravamen a las importaciones, julio de 1994 a septiembre de 1996

\begin{tabular}{lcc}
\hline & $\begin{array}{c}\text { Número de } \\
\text { productos }\end{array}$ & Por ciento \\
\hline $\begin{array}{c}\text { Productos considerados } \\
\text { Productos con variación } \\
\text { de tasas }\end{array}$ & 13428 & 100.0 \\
$\begin{array}{c}\text { Productos con dos o más } \\
\text { alteraciones de tasas }\end{array}$ & 3830 & 83.3 \\
$\begin{array}{c}\text { Productos con tres o más } \\
\text { alteraciones de lasas }\end{array}$ & 939 & 28.5 \\
$\begin{array}{c}\text { Productos con cinco o } \\
\text { más alteraciones de tasas }\end{array}$ & 148 & 1.1 \\
\hline
\end{tabular}

Fuente: Elaboración propia a partir de datos primarios.

3 Nótese que se trata de "variaciones" y no necesariamente de "reducciones". 
CUADRO 3

Brasil: Valor trimestral de las importaciones, tasas a las importaciones y número de productos afectados, 1994-1996

\begin{tabular}{|c|c|c|c|c|c|c|}
\hline \multirow[t]{2}{*}{ Período } & & \multirow{2}{*}{$\begin{array}{l}\text { Valor de las importaciones } \\
\text { (en millones de dólares cif) }\end{array}$} & \multirow{2}{*}{$\begin{array}{l}\text { Tasa simple } \\
\text { porcentual }\end{array}$} & \multicolumn{3}{|c|}{ Número de episodios de variación de las tasas } \\
\hline & & & & Reducciones & Aumentos & Total \\
\hline \multirow[t]{2}{*}{1994} & III & 7931 & 13.0 & 4738 & 21 & 4759 \\
\hline & IV & 11824 & 11.2 & 253 & 22 & 275 \\
\hline \multirow[t]{4}{*}{1995} & I & 12017 & 12.6 & 2462 & 5349 & 7811 \\
\hline & II & 13656 & 13.2 & 468 & 401 & 869 \\
\hline & III & 11916 & 13.2 & 84 & 85 & 169 \\
\hline & IV & 12030 & 13.3 & 63 & 97 & 160 \\
\hline \multirow[t]{3}{*}{1966} & I & 10737 & 13.1 & 1738 & 303 & 2041 \\
\hline & II & 12477 & 13.0 & 195 & 171 & 366 \\
\hline & III & 14965 & 13.2 & 13 & 150 & 163 \\
\hline
\end{tabular}

Fuente: Elaboración propia a partir de datos primarios.

GRÁFICO I

\section{Brasil: Tasas e importaciones}

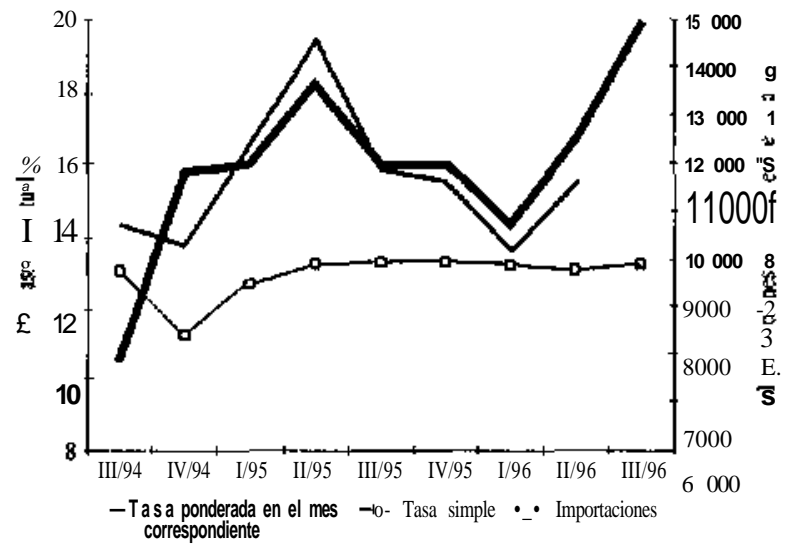

les siguió una evolución distinta: ese valor prácticamente se dobló entre el tercer trimestre de 1994 y el segundo trimestre de 1995, experimentó una leve caída en el trimestre siguiente y se mantuvo (con pequeñas variaciones) en ese nivel (en torno a los 12000 millones de dólares) desde entonces, con un nuevo repunte en el tercer trimestre de 1996.

Las últimas tres columnas del cuadro 3 ilustran algunas informaciones complementarias importantes. Con todo, antes de considerarlas cabe hacer una breve digresión metodológica para recalcar la importancia de incluir el indicador del número de variaciones de las tasas.

Si tomamos como ejemplo lo ocurrido entre diciembre de 1994 y enero de 1995, el cálculo de la tasa media simple del gravamen a las importaciones sugiere una situación de alza generalizada de las tasas, que subieron de $11.19 \%$ en la primera fecha a $12 ; 62 \%$ en la segunda Sin embargo, esas cifras son el resultado neto de una política que supuso simultáneamente 5093 alzas y 2691 reducciones de las tasas. En otras palabras, interesa que el análisis no se limite al ámbito más general, sino que considere también las informaciones a un nivel más detallado.

El cuadro 3 muestra claramente que las modificaciones de la estructura arancelaria se concentraban de hecho (a juzgar por el número de episodios de variación de las tasas) en el tercer trimestre de 1994 y en el primer trimestre de $1995,{ }^{4} \mathrm{y}$ que no era un proceso continuo.

Pese a que predomina el número de casos de reducción de tasas en el período en su conjunto, hubo también alzas arancelarias para un número importante de productos, en particular en el primer trimestre de 1995 (sobre todo para aquellos productos que tenían que adecuar su gravamen al Arancel Externo Común), y después (comienzos de 1996), aunque con una intensidad mucho menor, por razones de equilibrio de la balanza comercial.

El resultado general de esos movimientos - respecto a las tasas "simples"- - se muestra en el gráfico 2 .

Hay una clara tendencia a la reducción del arancel medio a fines de 1994, que representa en realidad la continuación de un proceso iniciado en 1990 (como se indica en el gráfico 2) y a una relativa estabilidad a partir del segundo trimestre de 1995, en torno a la mediana. La tasa más frecuente llegó a su punto mínimo en el primer trimestre de 1995 y se estabilizó a un nivel de $18 \%$ desde el segundo trimestre de ese año.

\footnotetext{
4 Con algún movimiento adicional en el primer trimestre de 1996.
} 
GRÁFICO 2

Brasil: Indicadores descriptivos de la política arancelaria reciente

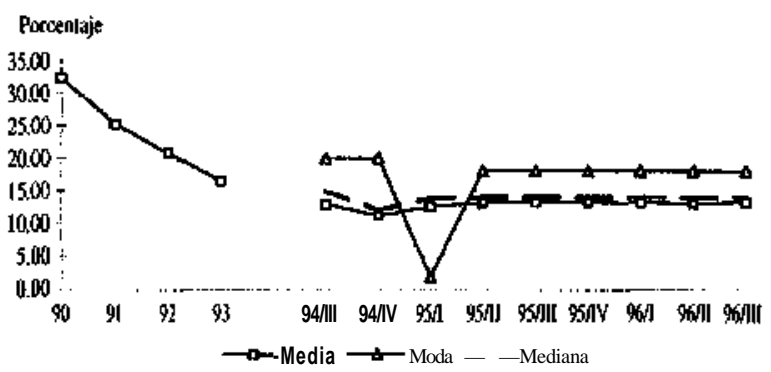

La teoría de la protección recomienda que, si es ineludible la adopción de aranceles a la importación, las distorsiones introducidas en el sistema económico serán tanto menores cuanto más bajas sean las tasas y menor su dispersión. En ese sentido, una reforma que reduzca los niveles arancelarios y que además disminuya la varianza de la distribución de tasas es un movimiento en la dirección que recomienda la teoría.

Sin embargo, el análisis de la evolución de la estructura arancelaria sugiere un comportamiento variado, a juzgar por la trayectoria del desvío-patrón de las tasas:

\begin{tabular}{lr}
\multicolumn{2}{c}{1994} \\
\hline III & IV \\
8.0 & 7.9
\end{tabular}

\begin{tabular}{crrr}
\multicolumn{4}{c}{1995} \\
\hline I & II & III & IV \\
7.2 & 9.4 & 9.6 & 9.8
\end{tabular}

\begin{tabular}{ccr}
\multicolumn{3}{c}{1996} \\
\hline I & II & III \\
9.0 & 8.4 & 8.9
\end{tabular}

Estas cifras indican que hubo inicialmente un movimiento hacia una mayor uniformidad de la estructura arancelaria (a tasas más bajas), hasta el primer trimestre de 1995, con una dispersión creciente en el resto de ese año, que fue seguida de una falta de convergencia en todo el año 1996.

\section{b) Las tasas ponderadas}

Hasta aquí los datos se han presentado sobre la base de tasas simples (es decir, medias ponderadas sólo por el plazo de vigencia) y en forma agregada. Sin embargo, se sabe que en ese período hubo cambios sustantivos en la lista de importaciones del país. A título ilustrativo, considérese que en 1993 las importaciones de bienes de consumo representaban $12 \%$ del valor total de las importaciones, y que esa participación subió en 1994 y 1995 a $17 \%$ y $22 \%$, respectivamente.

Esto obliga a considerar la estructura arancelaria en comparación con la gravitación efectiva de los productos afectados, o sea, hay que estimar las tasas del gravamen a las importaciones ponderadas por el valor de importación de cada producto.

La evolución excepcional de las corrientes comerciales en ese período plantea una dificultad adicional para identificar el vector de las importaciones más adecuado para proceder a esa ponderación. La estructura del período inmediatamente anterior no recoge las modificaciones ocurridas, y los años 1994 y 1995 se consideran atípicos, porque reflejaban estructuras de la demanda de productos importados muy influenciadas por la fase inicial de un proceso de estabilización.

La alternativa factible fue - como se describió en la sección anterior - ponderar el vector de las tasas simples por la composición de las importaciones en el período 1990-1993 como reflejo de un patrón mínimo, y por la estructura de las importaciones en 1995 como reflejo de uno máximo, complementando el análisis con las estimaciones de las tasas ponderadas por el valor corriente de las importaciones, o sea, del valor efectivamente registrado en cada trimestre.

El cuadro 4 y el gráfico 3 ilustran las diferencias en los criterios de ponderación.

Las tres estimaciones de tasas ponderadas muestran una fuerte caída del arancel en el último trimestre de 1994 y su recuperación en los dos trimestres siguientes, seguida de una nueva reducción durante el segundo semestre de 1995 y un leve aumento en 1996.

Del análisis de esos datos y del gráfico se desprende que: i) el comportamiento del arancel simple no es capaz de captar ese movimiento; ii) las modificaciones de la estructura de la lista de importaciones en el período fueron lo suficientemente pronunciadas como para conducir a resultados bastante distintos cuando consideramos para la ponderación la media del trienio anterior o un período de demanda acentuada como el año 1995; y iii) la ponderación por el valor de importación en el mismo trimestre lleva a resultados menos sesgados, con valores intermedios entre los resultados obtenidos con las otras dos ponderaciones. ${ }^{5}$

\footnotetext{
5 Obsérvese que los valores de las importaciones sólo responden en parte a los niveles de las tasas del gravamen a la importación: según la Secretaría de Ingresos Federales (datos publicados en la Gazeta Mercantil de 11/12/96), si se considera la relación entre el valor de las importaciones y el valor efectivamente pagado a título de gravámenes a las importaciones en los diez primeros meses de 1996, las importaciones brasileñas se efectuaron con un arancel medio real de $7.1 \%$, porcentaje que es bastante inferior a la tasa media nominal de $13.6 \%$ estimada por esa Secretaría. Eso se explica por la incidencia de los "regímenes especiales" que garantizan un tratamiento diferenciado a algunos productos importados. En términos sectoriales, ese mismo estudio señala diferencias manifiestas como, por ejemplo, para el material de transporte, cuyo arancel nominal es de $19.78 \%$ y cuyo gravamen real fue de $6.46 \%$ en 1996 , o el ítem de bienes de consumo con una tasa nominal de $24.38 \%$ y un arancel "real" de $11.12 \%$.
} 
CUADRO 4

Brasil: Tasas simples y ponderadas del gravamen a las importaciones, por trimestre, 1994-1996

\begin{tabular}{|c|c|c|c|c|c|}
\hline \multirow{2}{*}{\multicolumn{2}{|c|}{ Período }} & \multirow[t]{2}{*}{ Tasa simple porcentual } & \multirow{2}{*}{\multicolumn{3}{|c|}{ Tasa ponderada porcentual }} \\
\hline & & & & & \\
\hline 1994 & III & 12,95 & \multirow{2}{*}{$\begin{array}{c}\text { Media } \\
1990-1993 \\
13.45 \\
9.92\end{array}$} & $\begin{array}{c}\begin{array}{c}\text { Trimestre } \\
\text { corriente }\end{array} \\
14.36\end{array}$ & \multirow{2}{*}{$\begin{array}{c}\text { Trimestre } \\
\text { correspondiente en } 1995 \\
14.85 \\
14.02\end{array}$} \\
\hline & IV & 11.19 & & 13.68 & \\
\hline \multirow[t]{4}{*}{1995} & I & 12.62 & 13.04 & 16.57 & 16.57 \\
\hline & II & 13.18 & 13.74 & 19.46 & 19.46 \\
\hline & III & 13.22 & 13.49 & 15.79 & 15.79 \\
\hline & IV & 13.26 & 13.44 & 15.49 & 15.49 \\
\hline \multirow[t]{3}{*}{1996} & I & 13.13 & 12.70 & 13.51 & 18.39 \\
\hline & II & 12.99 & 12.86 & 15.47 & 19.72 \\
\hline & III & 13.19 & 13.03 & n.d, & 16.45 \\
\hline \multicolumn{6}{|c|}{$\begin{array}{l}\text { Media } \\
\text { total del período }\end{array}$} \\
\hline
\end{tabular}

Fuente: Elaboración propia a partir de datos primarios.

GRÁFICO 3

Brasil: Tasas ponderadas por las importaciones

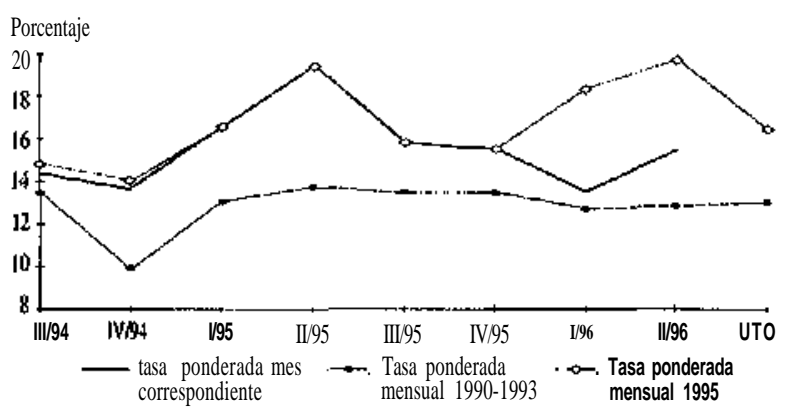

Esos resultados indican la necesidad de detallar el análisis para tomar en cuenta los movimientos dentro de cada trimestre - la relación entre el valor de las importaciones y la tasa del gravamen- así como la política relativa a determinados sectores.

Con todo, antes de proceder a ese detalle, caben algunas consideraciones respecto a la relación entre la estructura arancelaria nacional y el Arancel Externo Común acordado con los demás países miembros del Mercosur.

\section{El mito de la adopción anticipada del Arancel Externo Común (AEC)}

Se pensaba que Brasil adelantaría para septiembre de 1994 la adopción del Arancel Externo Común (AEC) acordado en el ámbito del Mercosur y previsto para entrar en vigor en enero de 1995, pero un análisis más detenido revela que esa afirmación merece algunos reparos.

Se atribuía a las resoluciones 506 y 507 del Ministerio de Hacienda, ambas fechadas en septiembre de 1994, el adelanto en tres meses de la entrada en vigor del AEC. En rigor, en ese mes se dictaron tres resoluciones importantes: la 492, que redujo a un nivel uniforme de $20 \%$ las tasas del derecho de importación para una serie de productos, y las 506 y 507, que modificaron -a niveles específicos- las tasas para una lista de productos.

No obstante, a fines de septiembre de 1994 y después de la entrada en vigor de las tres resoluciones, todavía había 124 productos con tasas superiores a $20 \%$; $^{6}$ no todos esos casos se referían a las excepciones previstas para el sector de la informática (sólo 48 productos de la posición 84.71) o las telecomunicaciones (sólo 6 productos de la posición 85.17),

Además, un examen del arancel brasileño a fines de septiembre de 1994 indicaba que existían: 2930 productos con gravámenes superiores; 5102 con aranceles inferiores; y 5396 con aranceles iguales a los niveles previstos para el AEC.

En otras palabras, a fines de septiembre la estructura del AEC se aplicaba sólo a $42 \%$ del total de productos de la lista de importaciones. Así pues, Brasil

6 Comparado con 452 productos en agosto de ese año. 
sólo adelantó la adopción del AEC con un ajuste parcial. ${ }^{7}$ La adopción del Arancel Externo Común y de la Nomenclatura Común sólo ocurrió efectivamente a partir del $1^{\circ}$ de enero de 1995 , en virtud del decreto $\mathrm{N}^{\circ}$ 1343. Esto no quiere decir que el esfuerzo de aproximación haya sido despreciable; sólo cabe destacar que no hubo una adopción anticipada completa.

Otra observación importante para entender la evolución de la estructura arancelaria brasileña en ese período está relacionada con las listas de excepción. Entre los meses de abril y diciembre de 1995, con la preocupación por reducir el nivel de precios internos mediante la competencia con las importaciones, se dictaron los decretos 1453, 1471, 1490, 1550, $1678 \mathrm{y}$ 1767, que establecían listas de productos sujetos a un tratamiento arancelario temporalmente diferenciado. ${ }^{8}$ Nuevamente, en abril de 1996 el decreto 1848 definió otra lista de excepciones.

En cuanto a los productos afectados, en enero de 1995 — fecha de adopción del AEC - 1272 productos constituían excepciones al AEC (anexo al decreto 1343), cifra que subió a 1700 productos en diciembre de ese año, por efecto de la dictación de los decretos señalados, que se consolidaron en el decreto 1767. En abril de 1996, el decreto 1848 consolida una nueva lista de excepciones de 1500 productos.

Considerando el conjunto de los movimientos efectuados en el período, en relación con el número de veces que variaron las tasas ( $\mathrm{y}$ no los productos afectados), podemos decir que para el período comprendido entre julio de 1994 y septiembre de 1996 ese número se distribuyó como sigue:

i) La adecuación parcial del arancel aduanero de Brasil al AEC en septiembre de 1994 se verificó en $23 \%$ de los casos, siendo todos de reducción de la tasa.

ii) La implantación del AEC en enero de 1995 implicó el $47 \%$ del total de variaciones del gravamen, con elevaciones en $32 \%$ de los casos y descensos en $15 \%$.

iii) La adopción de listas de excepción representó $23 \%$ del total, con reducciones en $16 \%$ de los casos y aumentos en $7 \%$.

\footnotetext{
7 Vinculado con reducciones de tasas más allá de los niveles previstos para el AEC, por razones de estabilización interna de precios.

${ }^{8}$ Las modificaciones introducidas durante 1995 pueden resumirse así: a) el decreto 1471 consolidó las excepciones al Arancel Externo Común (su anexo 1 corresponde a la lista de excepción nacional y los anexos 2 y 3 corresponden a la llamada lista Dallari); b) el decreto 1490 estableció el nuevo anexo 1 que revoca el anterior; c) el decreto 1550 corrigió e introdujo pequeñas modificaciones en el Arancel Externo Común; d) el decreto 1678 repitió el procedimiento anterior. Agradecemos a Honorio Kume por esas informaciones.
}

Esos datos ayudan a entender la trayectoria de la tasa media ponderada de las importaciones. En el gráfico 1 se observa un primer movimiento de reducción en el cuarto trimestre de 1994, lo que refleja el adelanto parcial del AEC (y el ajuste adicional de la estructura arancelaria), y luego un aumento cuando se adopta plenamente el arancel común, hasta llegar a un punto máximo en el segundo trimestre de 1995; hasta el primer trimestre de 1996, se reduce nuevamente en tres ocasiones, las que reflejan la importancia de las listas de excepciones, y vuelve a elevarse a partir de entonces.

El análisis de los datos mensuales sobre la tasa "simple" del impuesto a las importaciones y el valor de las importaciones (véase el gráfico 4) muestra también tres momentos:

i) En un primer momento —en julio y agosto de 1994 - el valor total de las importaciones comienza a aumentar, incluso antes de modificarse la estructura arancelaria: la explicación más probable está en el efecto-ingreso derivado del Plan Real, asociado a la valorización cambiaría.

ii) En un segundo período - entre septiembre y diciembre de 1994 — se reduce el nivel del arancel "simple". En ese período hay claramente un estímulo adicional a las importaciones, proporcionado por la variación de la tasa "simple" y por la permanencia de los efectos-ingreso y precio del período anterior.

iii) A partir de 1995 (sobre todo desde marzo) la tasa "simple" se estabiliza a un nivel de alrededor de $13 \%$ (al mismo tiempo que el tipo de cambio vuelve a variar). Sin embargo, persisten las fluctuaciones del

GRÁFICO 4

Brasil: Tasas e importaciones - valores mensuales

(Tasas porcentuales e importaciones en millones de dólares)

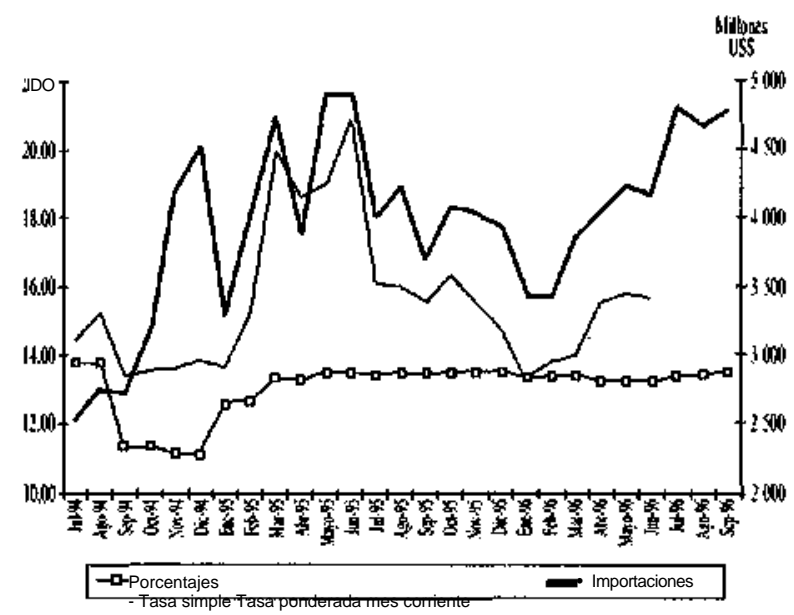


valor total de las importaciones. Lo que sugiere ese conjunto de datos es que a partir del segundo trimestre de 1995 la tasa "simple" pierde el poder de determinar las importaciones: su influencia parece limitarse al nivel del valor importado, pero no explica por cierto la variación del mismo, que pasa a depender de otros factores determinantes como la política cambiaría y otros.

El comportamiento de la tasa ponderada refleja esos tres momentos al elevarse entre julio y agosto de 1994 —incluso con una tasa "simple" constante-, mantenerse estable entre septiembre y enero de 1995 (la reducción de la tasa se compensa con el aumento de las importaciones), y a partir de entonces pasa a reflejar directamente las variaciones del valor de las importaciones.

Ese comportamiento sugiere que existen otros factores que hay que considerar. Entre ellos cabe destacar el análisis del comportamiento a nivel de los sectores más relevantes,

\section{Aranceles sectoriales}

Para el análisis sectorial se seleccionaron algunos capítulos (clasificación de dos dígitos de la Nomenclatura Brasileira de Mercadorias/Sistema Harmonizado (NBM/SH) de un total de 97 capítulos, sobre la base de dos criterios: i) número de variaciones de las tasas del gravamen a las importaciones en el período considerado, e ii) importancia relativa en el valor total de las importaciones.

Los 14 capítulos seleccionados conforme a esos criterios correspondieron en 1995 a $74 \%$ del valor total de las importaciones y a $67 \%$ del número de variaciones de las tasas en el período considerado. Es decir, esos capítulos concentraron la mayor parte de las variaciones de los derechos aplicados a las importaciones durante el período de julio de 1994 a septiembre de 1996. Dichos capítulos abarcan además 65\% del total de los productos (diez dígitos) afectados por modificaciones de tasas. ${ }^{9}$

\footnotetext{
${ }^{9}$ Ellos fueron: Cap. 11 - Productos de la industria molinera; Cap. 27 - Combustibles minerales, aceites minerales; Cap. 28 - Productos químicos inorgánicos; Cap. 29 - Productos químicos orgánicos; Cap. 39 - Plásticos y sus productos; Cap. 48 - Papel y cartón; Cap. 54 - Filamentos sintéticos o artificiales; Cap. 55 - Fibras sintéticas o artificiales; Cap. 72 - Hierro fundido, fierro y acero; Cap. 73 Obras de hierro fundido, fierro o acero; Cap. 84 - Máquinas, aparatos e instrumentos mecánicos; Cap. 85 - Material eléctrico y sus partes; Cap. 87 - Vehículos automotores y otros vehículos terrestres; Cap. 90 - Instrumentos y aparatos de óptica.
}

El cuadro 5 resume las informaciones básicas. Se observa en él que -igual que en el universo de la lista de importaciones- el número de reducciones de las tasas supera con creces al número de aumentos en el período. En esa muestra de 14 capítulos, en $59 \%$ de los casos hubo aumento de las tasas, una proporción menor que el $72 \%$ de los casos de reducción. Eso refleja la dispersión sectorial del proceso de liberalización comercial.

Las columnas cuarta a sexta del cuadro 5 muestran además que las tasas "simples" para esos 14 capítulos fueron siempre superiores a las tasas correspondientes al total de productos.

Esa relación se mantuvo en forma sistemática. El gráfico 5 muestra una comparación entre la trayectoria de las tasas de la muestra y las del total de los 97 capítulos durante el período considerado. Ambas presentan una reducción acentuada entre julio y diciembre de 1994, y una relativa estabilidad a partir de mayo de 1995. Sin embargo, la tasa "simple" para los 14 capítulos es sistemáticamente superior a la tasa correspondiente al total de productos, lo que explica la diferencia mencionada entre los porcentajes de participación de la muestra en los episodios de reducción y aumento de tasas.

Asimismo, como resultado de la política de importaciones, hubo durante el período considerado una relación directa y sistemática entre la tasa del gravamen a las importaciones y el valor de importación del producto (véase el cuadro 6 ). ${ }^{10}$

$\mathrm{El}$ análisis del cuadro 6 indica que los productos con mayor ponderación en el valor total de las importaciones estuvieron en promedio sujetos a tasas más elevadas. Hay una relación directa para cada tramo del valor de las importaciones.

Parte de la explicación de esos resultados debe buscarse en la política relativa a los sectores a que pertenecen esos productos. Los datos indican que las tasas más elevadas de ese conjunto de productos se dieron para los tejidos sintéticos, el calzado, los buses y los vehículos utilitarios, lo que sugiere preocupación

\footnotetext{
10 Ese cuadro muestra el nivel medio de las tasas "simples" en vigor durante el período considerado, por tramos de valor de las importaciones. Hay dos productos con un valor de importación sobre el millón de dólares en 1995: los vehículos de pasajeros y el petróleo. Como el régimen de importación del petróleo está totalmente regulado en el caso brasileño -siendo un producto con un mercado peculiar- y como la política de importación de automóviles presentó variaciones extremas durante el período, decidimos no incluir en el cuadro la información relativa a esos dos productos a fin de evitar distorsiones.
} 
CUADRO 5

Brasil: (Capítulos seleccionados según el número de variaciones de la tasa a las importaciones y/o la importancia relativa en el total de importaciones

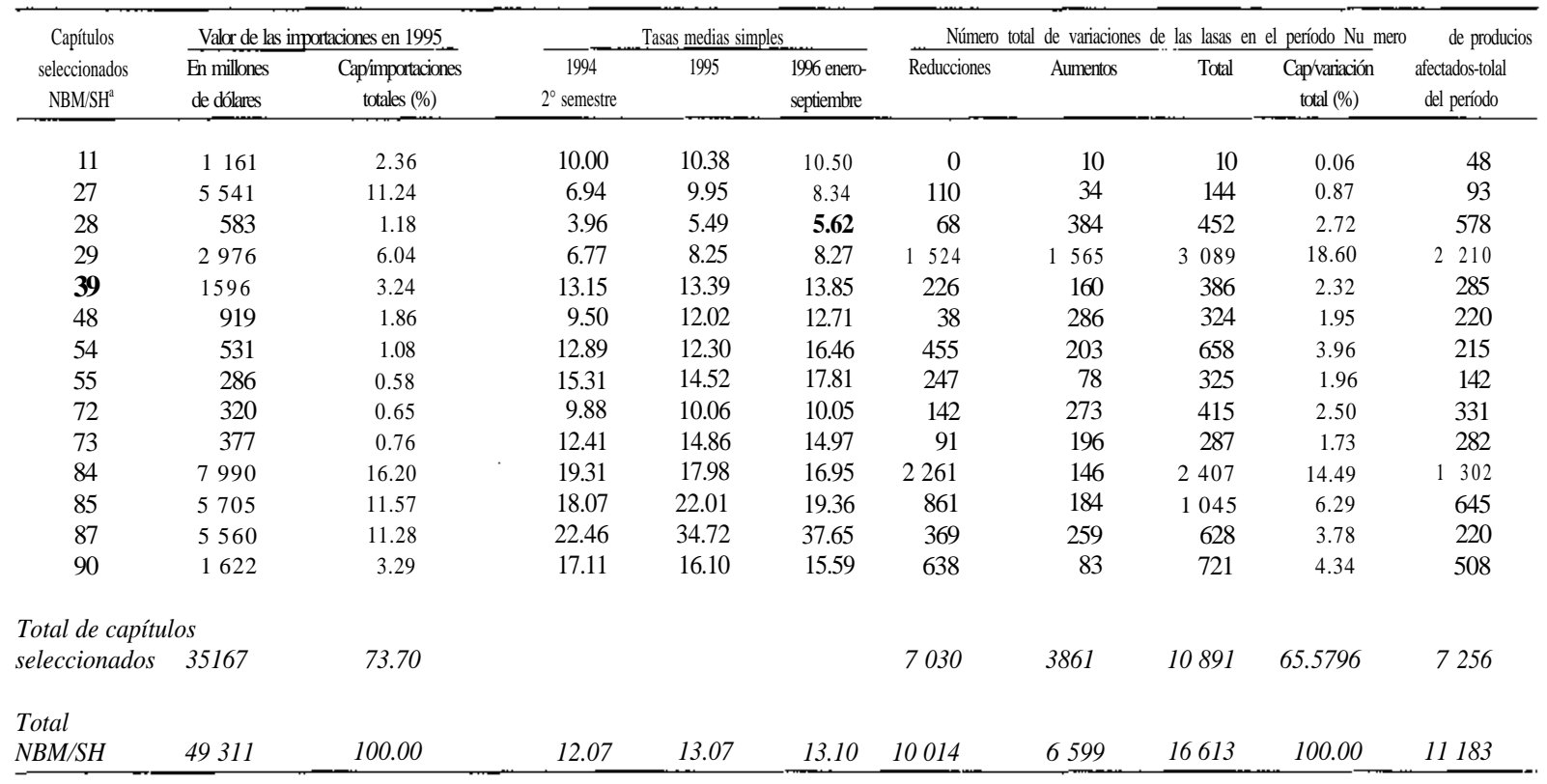

Fuente: Tabulación propia a partir de datos primarios.

a Nomenclatura Brasileira de Mercadorias / Sistema Harmonizado.

GRÁFICO 5

Brasil: Arancel medio simple, total y para capítulos seleccionados (En porcentajes)

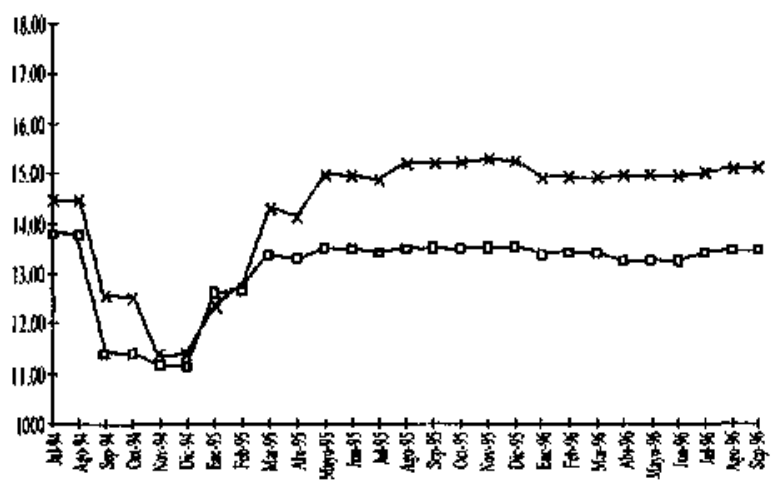

por los efectos de la importación sobre los productores nacionales.

Para todos los sectores considerados, los años entre 1990 y. 1993 son de reducción sostenida del derecho de importación. En algunos casos, esa reducción fue bastante notoria como, por ejemplo, para los productos de la industria molinera, en que el arancel se redujo de $28.8 \%$ en 1990 a $9.9 \%$ en 1993 . Otros ejemplos de reducción significativa son los plásticos y sus productos, máquinas, aparatos e instrumentos mecánicos, material eléctrico y sus partes y el sector productor de vehículos automotores y otros vehículos terrestres, en que el arancel bajó de $63.6 \%$ a $32.2 \%$ en ese período.

Los datos sectoriales en el período comprendido entre julio de 1994 y septiembre de 1996 muestran además que la trayectoria de las tasas "simples" es variada. ${ }^{11}$ Por ejemplo para los combustibles minerales, plásticos y sus productos, papel y cartón, productos químicos inorgánicos, productos químicos orgánicos y vehículos automotores, el derecho de importación llegó a su punto mínimo en el cuarto trimestre de 1994, mientras que para los filamentos sintéticos o artificiales y las fibras sintéticas o artificiales lo hizo en el primer trimestre de 1995. Respecto a las máqui-

11 Las posibles excepciones fueron las industrias molinera, los productos de hierro fundido, fierro $\mathrm{y}$ acero $\mathrm{y}$ ios instrumentos $\mathrm{y}$ aparatos de óptica, en que las variaciones de las tasas nominales fueron muy pequeñas. 
Brasil: Relación entre el valor de las importaciones y la tasa arancelaria aplicada

\begin{tabular}{lrrr}
\hline $\begin{array}{l}\text { Valor de las importaciones } \\
\text { (en millones de dólares) } \\
\text { por producto }\end{array}$ & Número de productos & $\begin{array}{c}\text { Participación porcentual } \\
\text { en el valor total de las } \\
\text { importaciones }\end{array}$ \\
\hline Sobre 20 & 369 & 56.97 & $\begin{array}{c}\text { Tasa "simple" } \\
\text { porcentual }\end{array}$ \\
Sobre 50 & 132 & 42.62 & 14.93 \\
Sobre 100 & 52 & 31.06 & 27.88 \\
Sobre 150 & $\mathbf{3 4}$ & 26.88 & 36.43 \\
\hline
\end{tabular}

Fuente: Tabulación propia a partir de datos primarios.

a Tasa media entre julio de 1994 y septiembre de 1996.

ñas, aparatos e instrumentos mecánicos, la trayectoria de las tasas "simples" muestra una reducción sistemática.

Lo que indican esas diferencias de trayectoria es que - a pesar de que los indicadores globales sugieren que el primer trimestre de 1995 fue el momento de mayor apertura de la economía - las diferentes evoluciones de las tasas sectoriales revelan a su vez que la política de apertura no obedeció a un movimiento uniforme generalizado, lo que también se aprecia en la evolución ya descrita de los desvíos-patrón de las tasas. En otras palabras, un período que se asocia con frecuencia con la apertura comercial abarca de hecho un conjunto variado de tratamientos sectoriales.

Un tercer aspecto que se desprende del análisis sectorial es el comportamiento de las tasas "simples" y ponderadas. En algunos sectores el peso de las importaciones de productos con tasas más altas que la media sectorial fue suficiente para elevar sistemáticamente los aranceles ponderados en comparación con los aranceles simples, como ocurrió con los combusti- bles minerales, los filamentos sintéticos (en 1994 y 1995) y los automóviles y otros vehículos terrestres. En cambio, para los productos químicos inorgánicos, plásticos y sus productos, papel y cartón, material eléctrico y sus partes e instrumentos y aparatos de óptica, sucedió lo contrario por el predominio de la lista de importación de productos con tasas más bajas. ${ }^{12}$

La teoría de la protección señala que, en algunas situaciones, el nivel arancelario establecido puede ser excesivo, dando lugar a lo que se ha convenido en llamar "aguar el arancel" (un margen que excede el nivel deseable de protección). Esa misma teoría señala que un nivel arancelario puede elevarse a tal punto de hacer inviable el comercio internacional.

La estimación de aranceles ponderados inferiores a los aranceles medios sectoriales puede ser un indicio de que -habida cuenta de la estructura de las importaciones y pese al proceso de apertura generalizada- en algunas industrias puede haber existido durante el período considerado cierto nivel de protección excesivo.

\section{III}

\section{Condicionantes de la política arancelaria}

\footnotetext{
12 Respecto a otros sectores — como los productos de la industria molinera, los productos químicos orgánicos, los productos de hierro fundido, fierro y acero, las máquinas y aparatos e instrumentos mecánicos- el grado de dispersión de las tasas para los diversos productos es reducido, por lo que la estructura de las tasas ponderadas es semejante a la de las tasas "simples"; dada la relativa homogeneidad de las tasas para los diversos productos en esos sectores, la estimación de la tasa ponderada no sufre la influencia del valor de importación que aparece tanto en el numerador como en el denominador de la fórmula de cálculo.
}

El primer aspecto que se destaca en el análisis de la política arancelaria en ese período es la influencia de la preocupación por estabilizar el nivel de precios internos —en un sentido amplio - en el diseño de esa política. Esa preocupación se refleja claramente tanto en la preparación de las listas de excepción ${ }^{13}$ como en

\footnotetext{
13 Obsérvese que fueron posteriores a la adopción del Arancel
} Externo Común. 
el diagnóstico subyacente a todo el proceso; se basa en la idea de que el acceso a los productos (sobre todo los insumos) a precios cercanos a los niveles internacionales contribuye a mejorar la eficiencia de todo el proceso productivo, la competitividad de la producción nacional y la sustentabilidad de la propia estabilización.

Los datos globales muestran un movimiento claro hacia una reducción general de las barreras al comercio, que tuvo como uno de sus resultados más significativos el aumento de! componente importado en la oferta interna: según estimaciones de Moreira y Correa (1996) la participación de las importaciones en el consumo aparente de la industria de transformación se habría elevado de $9.4 \%$ en 1993 a $15.5 \%$ en 1995 , sobresaliendo las de bienes de capital cuya participación subió de $28 \%$ a $42 \%$ en el mismo período. ${ }^{14}$

Igual importancia tuvo la influencia determinante que ejerció sobre la política aduanera la decisión política de consolidar el proceso de formación del Mercosur. Ese factor -sin precedente histórico en la política económica del país- afectó por cierto los resultados a fines de 1994 y comienzos de 1995.

Por último, ese período se caracteriza por una novedad en términos de la experiencia brasileña de las últimas décadas con respecto al proceso de determinación de las tasas del gravamen sobre la importación. Tradicionalmente, el arancel para las importaciones del Brasil — desde la ley de aranceles de 1957— se decidía sobre la base de soluciones negociadas entre los productores, los comercializadores y las autoridades del gobierno. Esa es una característica con pocos paralelos en otros países; cabe sostener que esa práctica puede haber contribuido a evitar las crisis observadas en otros países de América Latina, provocadas por el ritmo impuesto a las reformas comerciales. Sin embargo, también hay que reconocer que la influencia de intereses diversos muchas veces puede haber retardado el ritmo deseable (o económicamente recomendable) de las modificaciones del gravamen sobre las importaciones.

Comoquiera que se interprete su actuación, el hecho es que la existencia de una comisión de política aduanera integrada por representantes de diversos órganos de gobierno y del sector privado (con representantes de la agricultura, la industria y el comercio)

\footnotetext{
${ }^{14}$ Como advierten Moreira y Correa (1996), ello ocurrió sin que se observara un incremento correspondiente en los resultados de la exportación. Para la industria de transformación en su conjunto la relación exportación/producción se mantuvo casi constante: $14.5 \%$ en 1993 y apenas $14.9 \%$ en 1995 .
}

desempeñó un papel importante, ya que fomentó la transparencia y la definición mancomunada de criterios para modificar la estructura arancelaria. ${ }^{15}$

Ese mecanismo desapareció en 1990 — su ausencia es por tanto una peculiaridad del período estudiado- y !a definición de las tasas pasó a ser responsabilidad de un ministerio sectorial (industria) y del Ministerio de Hacienda.

Al respecto cabe señalar que la falta de un instrumento normativo como el descrito puede implicar costos. Por ejemplo, si se analiza la lasa del gravamen a la importación de algunos productos específicos a lo largo del tiempo se advierte que su evolución en ese período se aparta de la estabilidad recomendada por la teoría de la protección como un elemento importante para definir expectativas y, por ende, la gestión de los agentes económicos. El cuadro 7 ilustra algunos de esos casos.

La teoría de la protección sugiere que en las expectativas de los agentes económicos - tanto productores como consumidores- influye su esperanza de obtener utilidades a partir de una estructura arancelaria dada. Cuanto más homogénea sea esa estructura, menores serán los sesgos en términos de valor agregado, ${ }^{16} \mathrm{y}$, por tanto, menores también las distorsiones inducidas en la asignación de recursos. Asimismo, cuanto más estable sea esa estructura a lo largo del tiempo más dilatado será el horizonte de planificación por parte de los agentes, y mejor la eficiencia en la asignación de recursos. Los proyectos de mayor duración exigen reglas estables.

Sin embargo, los datos del cuadro 7 muestran que para diversos productos (en una clasificación de diez dígitos) el período comprendido entre julio de 1994 y septiembre de 1996 fue de inestabilidad, con una oscilación notoria de las tasas arancelarias para las importaciones. No existe una regla definida para saber cuál debe ser el intervalo de variación de un arancel por unidad de tiempo. No obstante, parece razonable suponer que los productores (así como los consumidores) de un bien que experimenta seis o más alteraciones de sus tasas en un período de 27 meses deben tener alguna dificultad para programar sus actividades con cierta precisión, sobre todo cuando esas variacio-

\footnotetext{
15 Para una descripción de las características, procedimientos, gestión y algunos resultados, véase Baumann y Morais (1988) y Baumann (1993).

16 Para el análisis de los niveles de protección efectiva en el perío-
} do reciente en Brasil, véase Kume (1996). 
CUADRO 7

Brasil: Algunos ejemplos de productos con cinco o más variaciones de tasas

\begin{tabular}{|c|c|c|c|}
\hline Capítulos & Nombre & Número de productos & Número de variaciones \\
\hline 15 & Aceites y grasas & 1 & 6 \\
\hline \multirow[t]{2}{*}{29} & Productos químicos orgánicos & 1 & 7 \\
\hline & & 6 & 5 \\
\hline 34 & Productos de limpieza & 12 & 6 \\
\hline \multirow[t]{2}{*}{54} & Filamentos sintéticos & 7 & 6 \\
\hline & & 5 & 7 \\
\hline 76 & Aluminio & 2 & 6 \\
\hline 83 & Productos de metales comunes & 1 & 6 \\
\hline \multirow[t]{2}{*}{84} & Máquinas y aparatos mecánicos & 1 & 6 \\
\hline & & 2 & 5 \\
\hline 85 & Máquinas, aparatos y material eléctrico & 11 & 5 \\
\hline 87 & Vehículos automotores & 61 & 5 \\
\hline
\end{tabular}

Fuente: Elaboración propia a partir de datos primarios.

CUADRO 8

Brasil: Ejemplos de variación de tasas para algunos productos

\begin{tabular}{|c|c|c|c|c|c|c|c|c|}
\hline \multirow{2}{*}{$\begin{array}{l}\text { Producto } \\
29.26 .90 .02 .00\end{array}$} & \multicolumn{8}{|c|}{ Evoluciôn del gravamen a las importaciones (en porcentaje) } \\
\hline & Adiponitrila & & & & & & & \\
\hline & $7 / 1994$ & 9/1994 & $12 / 1994$ & $5 / 1995$ & $11 / 1995$ & $2 / 1996$ & $4 / 1996$ & $8 / 1996$ \\
\hline & 15 & 14 & 2 & 4 & 8 & 10 & 2 & 12 \\
\hline \multirow[t]{3}{*}{34.01 .19 .03 .00} & Jabón industrial & & & & & & & \\
\hline & $7 / 1994$ & $1 / 1995$ & $5 / 1995$ & $11 / 1995$ & 2/1996 & 4/1996 & $8 / 1996$ & \\
\hline & 10 & 11 & 4 & 6 & 8 & 2 & 18 & \\
\hline \multirow[t]{3}{*}{$54.02 .49 .02 . .01$} & Tinturas de acrilico & & & & & & & \\
\hline & $7 / 1994$ & 9/1994 & 11/1994 & $4 / 1995$ & $5 / 1995$ & $2 / 1996$ & $4 / 1996$ & $8 / 1996$ \\
\hline & 20 & 16 & 2 & 0 & 6 & 10 & 6 & 16 \\
\hline \multirow[t]{3}{*}{54.02 .49 .04 .01} & Tinturas de poli]prop & ileno & & & & & & \\
\hline & $7 / 1994$ & 9/1994 & $11 / 1994$ & $4 / 1995$ & $5 / 1995$ & $2 / 1996$ & $4 / 1996$ & $8 / 1996$ \\
\hline & 20 & 16 & 2 & 0 & 6 & 10 & 6 & 16 \\
\hline \multirow[t]{3}{*}{84.22 .40 .99 .00} & Máquinas para emb & lar merc & & & & & & \\
\hline & & $7 / 1994$ & $11 / 1994$ & $1 / 1995$ & $6 / 1995$ & $7 / 1995$ & $1 / 1996$ & \\
\hline & & 20 & 0 & 19 & 0 & 19 & 18 & \\
\hline \multirow[t]{3}{*}{85.17 .10 .99 .00} & Aparatos telefónicos & & & & & & & \\
\hline & & 7/1994 & $1 / 1995$ & 3/1995 & $5 / 1995$ & $1 / 1996$ & 4/1996 & \\
\hline & & 30 & 19 & 70 & 63 & 56 & 30 & \\
\hline \multirow[t]{3}{*}{87.03} & Automóviles de pas & jeros & & & & & & \\
\hline & & $7 / 1994$ & 9/1994 & $1 / 1995$ & $2 / 1995$ & $1 / 1996$ & 4/1996 & \\
\hline & & 35 & 20 & 32 & 70 & 62 & 70 & \\
\hline
\end{tabular}

Fuente: Elaboración propia a partir de datos primarios.

nes se dan tanto para arriba como para abajo y son de gran magnitud.

El cuadro 8 muestra algunos ejemplos de productos en que esa oscilación fue bastante pronunciada y en que el proceso de determinación de las tasas del gravamen a las importaciones para algunos productos no siguió una orientación clara, ya sea en el sentido de una mayor apertura comercial o de establecer un sistema más autárquico. Tampoco es clara la lógica sectorial. Los productores de sectores tan dispares como la fabricación de productos de limpieza, máquinas, hilos y automóviles experimentaron notorias variaciones, sin dirección aparente: fluctuaciones que llegaron en algunos casos a diecinueve puntos porcen- 
tuales en uno u otro sentido en un intervalo de seis meses.

La explicación más probable de esos resultados parece ser que la política de importaciones buscó alcanzar objetivos múltiples, no siempre totalmente compatibles entre sí, como permitir el acceso ampliado a artículos importados y contribuir al programa de estabilización de precios, minimizando -en algunos casos- los costos para el productor nacional de esos bienes.

\section{IV}

\section{Resumen y consideraciones finales}

El presente artículo procuró analizar la evolución de los aranceles de importación en los primeros dos años de vigencia del Plan Real (julio de 1994 a septiembre de 1996), mediante el examen de toda la estructura de las tasas. El período de análisis correspondió a una intensificación decisiva del proceso de apertura comercial de la economía brasileña, iniciado en 1988 y que se intensificó a partir de 1990 con la definición de una trayectoria descendente de las tasas.

En ese período se registró un proceso de liberalización comercial - reflejado en el nivel medio de las tasas y en el grado de dispersión de su estructura-que llegó a su punto máximo en el primer trimestre de 1995. A partir de entonces hubo diversas alzas de tasas en forma diferenciada por sectores, lo que implicó una mayor dispersión de la estructura arancelaria. Los hechos señalados sugieren además que el nivel medio de las tasas nominales del gravamen a las importaciones se mantuvo relativamente constante a partir del segundo trimestre de 1995, con lo que el comportamiento del valor total de las importaciones estuvo determinado por otros elementos.

Ese proceso afectó prácticamente al $90 \%$ de la lista brasileña de importaciones y se dio en etapas asociadas a un proceso de apertura latu sensu, al adelanto parcial de la estructura del Arancel Externo Común del Mercosur y a la adopción de listas de excepción.

El análisis a nivel de sectores productivos revela - si hacemos abstracción de los casos puntuales del petróleo y los automóviles, que tuvieron un tratamiento diferenciado y un peso específico significativo en el conjunto de las importaciones- una relación directa entre el arancel nominal y el valor de importación por producto (lo que indica la preocupación por sectores productivos específicos), así como variaciones acentuadas durante el período en estudio. Además, el hecho de que para algunos sectores la tasa ponderada (por el valor de las importaciones) fuera inferior (durante meses) a la tasa media simple señala que existe un margen para reexaminar los niveles arancelarios en algunos casos.

Ese conjunto de observaciones permite formular algunas reflexiones sobre el diseño de la política de importaciones. En principio, ese diseño debería reflejar una función-objetivo clara para los agentes económicos a fin de que las decisiones de inversión pudieran basarse en el máximo de información. La claridad deriva no sólo de la identificación de propósitos sino también de la forma adoptada para alcanzar los objetivos. La experiencia brasileña es rica y singular en cuanto al funcionamiento de un cuerpo colegiado para cumplir con esa tarea. Hay argumentos favorables y contrarios a esa forma de operar, toda vez que la decisión relativa a bienes elaborados por unos pocos productores internos puede no ser eficiente si no es bien informada.

A su vez, la protección por sí misma no debía ser un objetivo ni mantenerse, sin tener en cuenta las alteraciones de las relaciones económicas básicas. Es decir, debería mantenerse cierto grado de flexibilidad para efectuar ajustes, ya sea adoptando una práctica común en otros países - la aplicación de mecanismos no arancelarios- $\mathrm{o}$ bien mediante alteraciones correspondientes del tipo de cambio. ${ }^{17}$ Sin embargo, la existencia de fluctuaciones del arancel nominal más allá de un nivel determinado en un plazo de tiempo relativamente corto ciertamente no es recomendable, incluso porque el arancel representa sólo una de las señales

\footnotetext{
17 Un método que caracterizó a la experiencia brasileña de las décadas de 1970 y 1980, pero cuyo uso debe considerarse con cautela dada la creciente importancia de las corrientes financieras externas para la economía brasileña y —en el período en cuestiónla lógica misma del proceso de estabilización que exige un tipo de cambio relativamente bajo y constante.
} 
(junto con el tipo de cambio y otros arbitrios) de los precios relevantes para los agentes involucrados y no siempre se identifica de inmediato el sentido de la variación. El ideal teórico es efectivamente una tasa única, uniforme y mantenida en el tiempo, lo que no siempre es viable. Una posibilidad que podría explorarse sería la delimitación de los márgenes de variación posible por períodos de tiempo como instrumento para reducir la inestabilidad excesiva que caracterizó el período analizado. 
El estudio tenía por objeto describir la política de definición de tasas del arancel sobre las importaciones en Brasil, en el período comprendido entre julio de 1994 y septiembre de 1996, y analizar fas estructuras derivadas. Por lo tanto, fue necesario trabajar con el universo de productos de la Nomenclatura Brasileira de Mercadorias, Sistema Harmonizado (NBM/SH-13 428 productos, en una clasificación de 10 dígitos) y examinar todos los documentos publicados en el Diario Oficial que determinaron modificaciones de esas tasas.

El segundo paso fue organizar y compatibilizar las informaciones sobre las tasas de importación de acuerdo con los datos del arancel aduanero brasileño (productos clasificados en 10 dígitos, según el sistema armonizado) y el arancel externo común (clasificación de ocho dígitos de la nomenclatura común del Mercosur). Dicho proceso se practicó a partir de la raíz común para cada producto, en una clasificación de seis dígitos, y utilizando la compatibilización elaborada en el arancel externo común y publicada en las ediciones aduaneras.

La tercera etapa del trabajo consistió en obtener las tasas vigentes en el mes de julio de 1994 para cada producto y en tabular — para cada mes subsiguiente - las alteraciones determinadas en resoluciones o decretos. De los 13428 productos hubo 11183 que experimentaron algún tipo de variación de la tasa, y 939 que experimentaron tres o más variaciones en ese período.

Sobre la base de esas informaciones se construyeron vectores de las tasas vigentes en cada mes para cada producto.

Para facilitar el análisis, los datos mensuales se agruparon en trimestres. El criterio básico para esa agrupación fue suponer que en cada trimestre la tasa representativa es aquella que estuvo en vigor por más tiempo. Fundados en ese supuesto, calculamos lo que denominamos la "tasa simple", que es de hecho la media de las tasas vigentes para cada producto ponderada por el plazo de vigencia de la tasa en cada trimestre.

Esa estimación nos permitió identificar los sectores más afectados y el número de incidencias de aumento y reducción de las tasas, además de posibilitar el cálculo de las estadísticas descriptivas genéricas de la media, moda y desvío-patrón de las tasas.

La evaluación de una estructura arancelaria requiere, no obstante, que la estimación de una tasa representativa refleje la importancia relativa de los diversos productos en términos de su participación en el valor total de las importaciones. Una estructura en que los aranceles estén ponderados por el valor de importación de cada producto tiene más significado económico que un vector de tasas nominales simples.

Eso plantea la dificultad de definir una estructura de importaciones que pueda utilizarse como referencia para la ponderación. En el período analizado influyeron notoriamente los efectos-ingreso y precio derivados del proceso de estabilización de los precios internos, además del efecto mismo sobre la demanda derivado del proceso de apertura comercial externa. Por consiguiente, la composición de las importaciones brasileñas en los años 1994 y 1995 fue muy distinta de la estructura de los años anteriores.

A fin de zanjar este problema consideramos tres estructuras alternativas de ponderación:

i) como "representativa" del período inmediatamente anterior a la adopción del Plan Real utilizamos la estructura de importaciones observada en 1990-1993 (la media de los valores de importación de cada producto);

ii) como referencia para someter a prueba los cambios sustantivos en la composición de las importaciones adoptamos la estructura de importaciones de 1995;

iii) suponemos que a) las variaciones de las tasas han contribuido a alterar el valor de importación, y al mismo tiempo y por efecto de a) para algunos productos, que b) las variaciones en el valor importado afectan las decisiones de alterar las tasas de los derechos de importación. Suponemos además (y en forma conscientemente arbitraria) que c) esos efectos tuvieron lugar con un desfase no superior a un trimestre. Ello dio origen a un tercer criterio de ponderación, en que las tasas relativas a un trimestre dado son ponderadas por el valor corriente de las importaciones en el mismo trimestre.

Se tuvo especial cuidado en la tabulación de los datos relativos al cuarto trimestre de 1994, que es considerado por diversos analistas como el período de mayor intensificación del proceso de apertura comercial. Como disponíamos de toda la reglamentación muy detallada, fue posible seguir de cerca, entre otros aspectos, la relación efectiva entre la estructura arancelaria brasileña y el arancel externo común, formulando reservas a algunas conclusiones halladas con frecuencia en los estudios sobre ese período.

Los datos sobre las tasas del gravamen a las importaciones fueron recopilados a partir del análisis de las resoluciones y decretos publicados en el Diario Oficial de la Unión durante el período considerado, así como de la recopilación de leyes de comercio exterior publicada por las ediciones aduaneras.

Los datos sobre el valor de importación mensual de cada producto se obtuvieron directamente del procesamiento primario efectuado por la Secretaria de Ingresos Federales, lo que permitió calcular las tasas ponderadas (por valor y por período de vigencia) y agrupar los datos por capítulo (dos dígitos del sistema armonizado) y por subperíodos.' 
ANEXO 2

Brasil: Tasas nominales porcentuales del gravamen a las importaciones en sectores seleccionados, 1990-1996

\begin{tabular}{|c|c|c|c|c|c|c|c|c|c|c|c|c|c|c|}
\hline Período & Cap. 11 & Cap.27 & Cap. $2 S_{i}$ & Cap. 29 & Cap. 39 & Cap. 48 & Cap. 54 & Cap. 55 & Cap. 72 & Cap. 73 & Cap. 84 & Cap. 85 & Cap. 87 & Cap. 90 \\
\hline 1990 & 28.75 & 5.00 & 13.55 & 24.21 & 32.26 & 26.31 & 25.53 & 30.00 & 21.99 & 26.95 & 36.56 & 38.89 & 63.57 & 29,97 \\
\hline 1991 & 21.15 & 1.49 & 7.70 & 17.12 & 25.91 & 15.09 & 24.37 & 30.00 & 16.43 & 22.73 & 28.90 & 31.79 & 48.72 & 25.63 \\
\hline 1992 & 12.08 & 0.53 & 7.07 & 15.69 & 21,86 & 12.04 & 21,17 & 25.00 & 13.98 & 18.78 & 24.72 & 26.84 & 40.63 & 21.24 \\
\hline 1993 & 9.90 & 0.57 & 5.10 & 13.03 & 16.28 & 10.49 & 21.21 & 24,26 & 11.09 & 17.29 & 20.29 & 22.11 & 32.18 & 19.34 \\
\hline 1994 & 9.48 & 0.41 & 4,52 & 12.30 & 14.74 & 10.49 & 18.06 & 19.07 & 10.21 & 13.35 & 19.37 & 19.01 & .27 .39 & 17.77 \\
\hline 1995 & 10.40 & 9.95 & 5.49 & 8.25 & 13.39 & 12.02 & 12.30 & 14.52 & 10.06 & 14.86 & 17.98 & 22.01 & 34.72 & 16.10 \\
\hline 1996 (ene/set) & 10.50 & 8.34 & 5.62 & 8.27 & 13.85 & 12.71 & 16,46 & 17.81 & 10.05 & 14.97 & 16.95 & 19.36 & 37.65 & 15.59 \\
\hline 1994 III & 10.00 & 7.24 & 4.25 & 9.41 & 14,03 & 9.50 & 16.37 & 18.06 & 10.22 & 12.78 & 19.37 & 18.34 & 24.99 & 17.45 \\
\hline 1994 IV & 10.00 & 6.65 & 3.66 & 4.13 & 12.28 & 9.50 & 9.40 & 12,55 & 9.54 & 12.04 & 19.24 & 17.80 & 19.93 & 16,77 \\
\hline 19951 & 10.38 & 9.95 & 5.44 & 8.23 & 13.24 & 12.73 & 7.13 & 10.38 & 10.66 & 14.92 & 17.63 & 18.70 & 26.52 & 16.28 \\
\hline 1995 II & 10.38 & 9.95 & 5.46 & 8.26 & 13.35 & 12.02 & 10.76 & 13.55 & 9.95 & 14.80 & 18.34 & 23.52 & 37,49 & 16.28 \\
\hline 1995 III & 10.38 & 9.96 & 5.48 & 8.27 & 13.52 & 11.67 & 15.04 & 16.59 & 9.68 & 14.84 & 17.97 & 22.90 & 37.44 & 16.10 \\
\hline $1995 \mathrm{IV}$ & 10,38 & 9.96 & 5.57 & 8.26 & 13.46 & 11.67 & 16.26 & 17.57 & 9.95 & 14.87 & 17.97 & 22.90 & 37.44 & 15.74 \\
\hline $1996 \mathrm{I}$ & 10.50 & 8.32 & 5.60 & 8.27 & 13.85 & 12.46 & 16,42 & 17.81 & 10.04 & 14,97 & 17.29 & 21.78 & 34.25 & 15.47 \\
\hline 1996 II & 10.50 & 8.35 & 5.60 & 8.27 & 13.85 & 12.46 & 16.34 & 17.81 & 10.04 & 14.97 & 16.80 & 18.16 & 39.05 & 15.67 \\
\hline $1996 \mathrm{III}$ & 10.50 & 8.35 & 5.65 & 8.27 & 13.85 & 13.21 & 16.62 & 17.81 & 10.05 & 14.97 & 16,78 & 18.13 & 39.64 & 15,64 \\
\hline
\end{tabular}

Fuente: Tabulación de datos primanos (véase el texto).

Cap. 11 - Productos de la industria molinera; Cap. 27 - Combustibles minerales, aceites minerales; Cap. 28 Productos químicos inorgánicos; Cap. 29 - Productos químicos orgánicos; Cap. 39 - Plásticos y sus obras; Cap. 48 - Papel y cartón; Cap. 54 - Filamentos sintéticos o artificiales; Cap. 55 - Fibras sintéticas o artificiales; Cap. 72 - Hierro fundido, fierro y acero; Cap. 73 - Obras de hierro fundido, fierro o acero; Cap, 84 - Máquinas, aparatos e instrumentos mecánicos; Cap. 85 - Material eléctrico y sus partes; Cap. 87 - Vehículos automotores y otros vehículos terrestres; Cap. 90 - Instrumentos y aparatos de óptica.

\section{Bibliografía}

Baumann, R. (1993); A Political Economy Analysis of Import Tariff Policy in Brazil: 1980-1988, Serie reformas de política pública, $\mathrm{N}^{\circ}$ 3, Santiago de Chile, Comisión Económica para América Latina y el Caribe (CEPAL).

Baumann, R. y J.M. Morais (1988): A economía política da protecáo no Brasil e a Rodada Uruguai, Texto para discussão, $\mathrm{N}^{\circ} 2$, Rio de Janeiro, Instituto de Pesquisa Econỏmica Aplicada (IPEA), septiembre.

Kume, H, (1996): A política de importaçäo no plano real e a estrutura de protecáo efetiva, Texto para discussao, $\mathrm{N}^{\circ} 423$, Rio de Janeiro, IPEA, mayo.

Moreira, M.M. y P.G. Correa (1996): Abertura comercial e industria: $o$ que se pode esperar e o que se vem obtendo, Texto para discussão $\mathrm{N}^{\circ} 49$, Rio de Janeiro, Banco Nacional de Desarrollo Económico y Social /Área de Planejamento/Banco Central do Brasil, Departamento Econổmico.

Nonnemberg, M, (1996): Impacto dos financia/tientos sobre o crescimento das importacoes brasileiras: 1992/95, Texto para discussão $\mathrm{N}^{\circ} 432$, Rio de Janeiro, IPEA, agosto.

Pinheiro, A.C. y G. Bacha de Almeida (1995): Padroes setoriais da protecāo na economía brasileira, E. Teixeira y D. Aguiar (eds.), Comercio internacional e comercializacáo agrícola, Vicosa, Fondo para el Financiamiento de Estudios y Proyectos/ Fundaçăo de Amparo a Pesquisa do Estado da Minas Gerais.

(Traducido del portugués) 